\title{
miR-144 inhibits growth and metastasis of cervical cancer cells by targeting VEGFA and VEGFC
}

\author{
PINGPING TAO ${ }^{1 *}$, HAO WEN ${ }^{2,3^{*}}$, BINLIE YANG ${ }^{1}$, AI ZHANG ${ }^{1}$, XIAOHUA WU ${ }^{2,3}$ and QING LI $^{4}$ \\ ${ }^{1}$ Department of Obstetrics and Gynecology, People's Hospital of Shanghai Pudong New Area, \\ Shanghai 201299; ${ }^{2}$ Department of Gynecological Oncology, Fudan University Shanghai Cancer Center; \\ ${ }^{3}$ Department of Oncology, Shanghai Medical College, Fudan University, Shanghai 200032; ${ }^{4}$ Department \\ of Pathology, People's Hospital of Shanghai Pudong New Area, Shanghai 201299, P.R. China
}

Received September 27, 2016; Accepted July 11, 2017

DOI: $10.3892 /$ etm.2017.5392

\begin{abstract}
MicroRNAs (miRs) are aberrantly expressed in various cancer types and have critical roles in their genesis and progression. miR-144 has been identified to be involved in the development of hepatocellular carcinoma and rectal cancer. However, the roles of miR-144 in cervical cancer and the underlying molecular mechanisms have remained elusive. The present study identified that miR-144 was significantly decreased in cervical cancer tissues compared with that in matched normal cervical tissues as well as in metastatic vs. non-metastatic cervical cancer tissues. miR-144 downregulation was significantly associated with the International Federation of Gynecology and Obstetrics stage and lymph node metastasis. In a gain-of function study, miR-144 mimics were transfected into the Hela and C33A cervical cancer cell lines, which led to suppression of cell growth. In addition, overexpression of miR-144 inhibited the migration and invasion of Hela and C33A cells. Furthermore, a bioinformatics analysis identified vascular endothelial growth factor A (VEGFA) VEGFC as two novel target genes of miR-144. Of note, a dual luciferase reporter assay, reverse-transcription quantitative polymerase chain reaction analysis and western blot analysis demonstrated that miR-144 repressed the expression of VEGFA and VEGFC by directly targeting to their 3'-untranslated region. Taken together, the results suggested that miR-144 acts as a tumor suppressor in the proliferation and metastasis of cervical cancer cells by directly targeting VEGFA and VEGFC, suggesting that miR-144 may be a novel promising diagnostic and therapeutic biomarker for cervical cancer.
\end{abstract}

Correspondence to: $\mathrm{Dr}$ Xiaohua $\mathrm{Wu}$, Department of Gynecological Oncology, Fudan University Shanghai Cancer Center, 270 Dongan Road, Shanghai 200032, P.R. China

E-mail:wu2005md@sina.com

*Contributed equally

Key words: microRNAs, cervical cancer, microRNA-144, vascular endothelial growth factor A, vascular endothelial growth factor C, suppressor

\section{Introduction}

Cervical cancer is the second most frequent gynecological cancer type worldwide and accounts for almost $12 \%$ of all malignancies in women $(1,2)$. Cervical cancer is the result of a multistep process involving the transformation of normal cervical epithelium to a cervical intraepithelial pre-neoplasm, which is subsequently transformed into cervical cancer (3). Infection with high-risk human papillomaviruses (HPVs) has an important role in the initiation of cervical cancer $(4,5)$. However, increasing evidence has indicated that HPV infection alone is insufficient for the development and progression of cervical cancer (5). Therefore, there is a compelling requirement for understanding of the other molecular mechanisms underlying the process of the genesis and progression of the malignant phenotype.

MicroRNAs (miRNAs/miRs) are a class of endogenous, small non-coding RNAs that are comprised of 18-25 nucleotides and control gene expression via binding with complementary sites in the 3'-untranslated regions (UTRs) of mRNAs $(6,7)$. Accumulating evidence demonstrated that certain miRNAs are deregulated in a number of human cancer types and are involved in the regulation of numerous biological processes, including cell growth, differentiation, invasion, migration and apoptosis $(8,9)$. miRNAs act as tumor suppressors or oncogenes in cancer depending on the nature of their target genes $(10,11)$. Certain miRNAs have been demonstrated to be involved in the genesis and progression of cervical cancer (9). For instance, miR-328 suppresses cervical cancer cell proliferation and tumorigenesis in vitro and in vivo by targeting transcription factor 7 like 2 expression (12). Furthermore, miR-486-3p targeting extracellular matrix protein 1 inhibits cell proliferation and metastasis in cervical cancer (13). miR-519d contributes to the progression and metastasis of cervical cancer by directly regulating $\mathrm{Smad} 7$ (14). miR-200b was demonstrated to suppress cell invasion and metastasis by suppressing epithelial-mesenchymal transition in cervical carcinoma (15).

miR-144 was originally identified to be an erythroid-specific miRNA, which is essential for the survival and maturation of the erythroid lineage $(16,17)$. Ample studies have also reported that miR-144 acts as a tumor suppressor in different types of human cancer, including hepatocellular carcinoma (18), rectal cancer (19) and B-cell lymphoma (20). A recent miRNA 
microarray analysis demonstrated that miR-144 expression was significantly downregulated in patients with cervical cancer and lymph node metastasis as compared with those without lymph node metastasis (21). However, the functional role of miR-144 in cervical cancer has remained to be elucidated.

The present study investigated the expression of miR-144 in cervical cancer tissues and explored its potential effects on the growth, migration and invasion of cervical cancer cells. In addition, vascular endothelial growth factor A (VEGFA) and VEGFC were identified as targets of miR-144 in cervical cancer cells. These findings indicated a novel molecular mechanism underlying in the regulatory roles of miR-144 in cervical cancer. Thus, miR-144 may be a promising therapeutic tool for cervical cancer in the future.

\section{Material and methods}

Cell lines and culture. The HeLa and C33A cervical cancer cell lines were obtained from the Cell Bank of the Chinese Academy of Sciences (Shanghai, China). Cells were cultured in RPMI1640 (Gibco; Thermo Fisher Scientific, Inc., Waltham MA, USA) supplemented with $10 \%$ fetal bovine serum (FBS; Gibco; Thermo Fisher Scientific, Inc.), $100 \mathrm{~g} / \mathrm{ml}$ streptomycin sulfate and $100 \mathrm{U} / \mathrm{ml}$ penicillin sodium in a humidified incubator with $5 \% \mathrm{CO}_{2}$ at $37^{\circ} \mathrm{C}$. Cells in the logarithmic growth phase were used for further study.

Oligonucleotide transfection. miR-144 mimics and scrambled oligonucleotide as the negative control (NC) were chemically synthesized and purchased from Shanghai GenePharma (Shanghai, China). miR-144 mimics or NC (100 nM) in $2 \mathrm{ml}$ RPMI1640 medium were transfected into HeLa and C33A cells with Lipofectamine 2000 reagent (Invitrogen; Thermo Fisher Scientific, Inc.) after the cells reached $60-80 \%$ confluence, according to the manufacturer's protocol. After $6 \mathrm{~h}$ of transfection, the medium was replaced with RPMI1640 containing $10 \%$ FBS. The cells were collected $48 \mathrm{~h}$ later, and the expression of miR-144 in the cervical cancer cell lines was detected by Reverse-transcription quantitative polymerase chain reaction analysis (RT-qPCR).

Clinical samples of cervical cancer. A total of 35 cervical cancer tissues and matched normal tissues were collected from the Department of Gynecologic Tumor at Fudan University Shanghai Cancer Center (Shanghai, China) from January 2010 to December 2014. The tissues that were obtained from surgery were immediately frozen in liquid nitrogen and subsequently stored at $-80^{\circ} \mathrm{C}$ for further study. Pathological classification and staging were performed according to the International Federation of Gynecology and Obstetrics (FIGO) criteria (22). Based on lymph node metastasis, 35 patients with cervical cancer were divided into two groups, including patients with lymph node metastasis $(n=9)$ and those with no lymph node metastasis $(n=26)$. All patients provided their written informed consent to be enrolled in the study. The present study was approved by the Ethics Committee of Fudan University Shanghai Cancer Center (Shanghai, China) and experiments were in accordance with the Declaration of Helsinki. The characteristics of the cervical cancer patients are displayed in Table I.
Table I. Association between miR-144 expression and clinicopathological characteristics.

\begin{tabular}{|c|c|c|c|c|}
\hline \multirow[b]{2}{*}{ Variable } & \multirow[b]{2}{*}{ Cases (n) } & \multicolumn{2}{|c|}{$\begin{array}{l}\text { miR-144 } \\
\text { expression }\end{array}$} & \multirow[b]{2}{*}{ P-value } \\
\hline & & Low & High & \\
\hline Age (years) & & & & 0.259 \\
\hline$<45$ & 22 & 14 & 8 & \\
\hline$\geq 45$ & 13 & 11 & 2 & \\
\hline Tumor size & & & & 0.723 \\
\hline$\leq 4 \mathrm{~cm}$ & 19 & 13 & 6 & \\
\hline$>4 \mathrm{~cm}$ & 16 & 12 & 4 & \\
\hline Histological type & & & & 0.661 \\
\hline Squamous cell carcinoma & 27 & 20 & 7 & \\
\hline Adenocarcinoma & 8 & 5 & 3 & \\
\hline Histological differentiation & & & & 0.266 \\
\hline Well/moderate & 15 & 9 & 6 & \\
\hline Poor & 20 & 16 & 4 & \\
\hline FIGO stage & & & & $0.004^{\mathrm{a}}$ \\
\hline IB & 24 & 21 & 3 & \\
\hline$>\mathrm{IB}$ & 11 & 4 & 7 & \\
\hline Lymph node metastasis & & & & $0.007^{\mathrm{a}}$ \\
\hline No & 26 & 22 & 4 & \\
\hline Yes & 9 & 3 & 6 & \\
\hline
\end{tabular}

${ }^{\mathrm{a}} \mathrm{P}<0.05$. FIGO, International Federation of Gynecology and Obstetrics; miR, microRNA.

$R T-q P C R$. Total RNA was extracted from cervical cancer tissues and cells by using TRIzol reagent (Invitrogen; Thermo Fisher Scientific, Inc.) according to the manufacturer's protocol. For detection of miR-144 expression, RT was performed by using a Super-Script III RT kit (cat. no. 18080085; Invitrogen; Thermo Fisher Scientific, Inc.). To determine VEGFA and VEGFC expression, mRNA was converted into complementary (c)DNA by using a PrimeScript RT reagent kit (cat. no. RR047A; Takara, Tokyo, Japan). qPCR was performed by using a SYBR ${ }^{\circledR}$ Premix Ex Taq ${ }^{\mathrm{TM}}$ II kit (cat. no. RR036A; Takara) with analysis in an ABI 7500 Real-Time PCR System (Applied Biosystems; Thermo Fisher Scientific, Inc.). The qPCR conditions were $95^{\circ} \mathrm{C}$ for 5 min followed by 40 cycles of denaturation at $95^{\circ} \mathrm{C}$ for $10 \mathrm{sec}$ and annealing/elongation at $60^{\circ} \mathrm{C}$ for $30 \mathrm{sec}$. U6 small nuclear RNA and GAPDH were assessed as endogenous controls. Primer sequences were as follows: miR-144, forward, 5'-TCATGTAGTAGATATGAC AT-3' and reverse miscript universal primer (Qiagen $\mathrm{GmbH}$, Hilden, Germany); U6 forward, 5'-CTCGCTTCGGCAGCA CA-3' and reverse, 5'-AACGCTTCACGAATTTGCGT-3'; VEGFA forward, 5'-TTTCTGCTGTCTTGGGTGCAT TGG-3' and reverse, 5'-ACCACTTCGTGATGATTCTGC CCT-3'; VEGFC forward, 5'-GAGGAGCAGTTACGGTCT GTG-3' and reverse, 5'-TCCTTTCCTTAGCTGACACTT GT-3'; GAPDH forward, 5'-CTGGGCTACACTGAGCACC-3' and reverse, 5'-AAGTGGTCGTTGAGGGCAATG-3'. The RT-qPCR was performed using the $2^{-\Delta \Delta \mathrm{Cq}}$ method (23). 
MTT assay. The viability of the HeLa and C33A cells was evaluated by using the MTT method. The cells $\left(5 \times 10^{3}\right.$ cells/well) were seeded and cultured in 96-well plates, and MTT solution (Sigma-Aldrich; Merck KGaA, Darmstadt, Germany) was added to a final concentration of $0.5 \mathrm{mg} / \mathrm{ml}$ at $48 \mathrm{~h}$ after transfection. The cells were incubated for another $4 \mathrm{~h}$ at $37^{\circ} \mathrm{C}$ with $5 \% \mathrm{CO}_{2}$. Subsequently, the medium was removed and $150 \mu \mathrm{l}$ of dimethylsulfoxide (Sigma-Aldrich; Merck KGaA) was added to each well to dissolve the formazan crystals. Finally, the optical density at $480 \mathrm{~nm}$ was measured by using an ELX-800 Microplate Reader (BioTeke, Winooski, VT, USA).

Migration and invasion assay. A wound healing assay was performed to assess the migration ability of cervical cancer cells. In brief, cells were seeded in 6-well plates at a density of $10^{6}$ cells/well and cultured to confluence. Cell monolayers were scraped with a sterile 10- $\mu$ l pipette tips (EMD Millipore, Billerica, MA, USA) to generate scratch wounds and washed three times with PBS (Sigma-Aldrich; Merck KGaA) to remove cell debris. The cells were incubated at $37^{\circ} \mathrm{C}$ with $5 \%$ $\mathrm{CO}_{2}$ for another $24 \mathrm{~h}$. To determine the migration distances, wounds were observed under a CX41 inverted microscope (Olympus, Tokyo, Japan) at x100 magnification and images were captured.

A cell invasion assay was performed by using Transwell ${ }^{\circledR}$ chambers (BD Biosciences, Franklin Lakes, NJ, USA). In brief, $300 \mu \mathrm{l}$ cell suspension was added to the upper chamber pre-coated with Matrigel ${ }^{\mathrm{TM}}$ (BD Biosciences) and $500 \mu \mathrm{l}$ RPMI1640 with $10 \%$ FBS as the chemoattractant was added to the lower chambers. The cells were incubated at $37^{\circ} \mathrm{C}$ with $5 \%$ $\mathrm{CO}_{2}$ for $24 \mathrm{~h}$. Following careful removal of cells remaining on the upper surface of the membrane, those on the lower surface of the membrane were fixed in pure methanol (Sigma-Aldrich; Merck KGaA) for 20 min and stained with $0.1 \%$ crystal violet (Sigma-Aldrich; Merck KGaA) for 15 min. Stained cells were visualized and counted in five randomly selected fields under a CX41 inverted microscope at x200 magnification.

Dual luciferase reporter assay. The full-length 3'-UTRs of VEGFA and VEGFA [wild type (WT)] were amplified by PCR from cDNA and cloned into the PGL3-Basic Vector (Promega, Madison, WI, USA). The mutant (MUT) construct of VEGFA and VEGFA 3'-UTRs was generated by using a QuickChange Site-Directed Mutagenesis kit (cat. no. R407; Takara). Co-transfection of WT or MUT VEGFA or VEGFA reporter vector with miR-144 mimic or $\mathrm{NC}$ was performed by using Lipofectamine 2000. After $48 \mathrm{~h}$ of transfection, dual luciferase activity was measured by using a dual luciferase reporter assay system (Promega) according to the manufacturer's protocols.

Protein extraction and western blot analysis. Total protein was extracted from the cultured cells by using radioimmunoprecipitation assay buffer (Invitrogen; Thermo Fisher Scientific, Inc.) at $4^{\circ} \mathrm{C}$. The total protein concentration was determined by an Enhanced BCA Protein Assay kit (Beyotime Institute of Biotechnology, Haimen, China). Equal amounts of proteins $(40 \mu \mathrm{g})$ were separated by $8-12 \%$ SDS-PAGE and transferred onto a polyvinylidene difluoride membrane (EMD Millipore). The membrane was incubated with Tris-buffered saline with
Tween-20 containing $5 \%$ non-fat milk for $2 \mathrm{~h}$ at $37^{\circ} \mathrm{C}$. The membrane was incubated with mouse anti-human monoclonal anti-VEGFA (dilution, 1:500; cat. no. ab1316; Abcam, Cambridge, MA, USA), anti-VEGFC (dilution, 1:1,000; cat. no. ab191274; Abcam) and anti-GAPDH (dilution, 1:2,000; cat. no. ab9482; Abcam) antibodies at $4^{\circ} \mathrm{C}$ overnight, followed by incubation with horseradish peroxidase-conjugated secondary antibodies (1:2,000; cat. no. A21010; WuHan AmyJet Scientific Inc., Wuhan, China) at room temperature for $1 \mathrm{~h}$. Blots were visualized by an enhanced chemiluminescence kit (Pierce; Thermo Fisher Scientific, Inc., Rockford, IL, USA).

Statistical analysis. Statistical analysis was performed with SPSS statistical software, version 17.0 (SPSS, Inc., Chicago, IL, USA). Values are expressed as the mean \pm standard deviation of at least three independent experiments. Differences between two groups were analyzed by a two-tailed Student's t-test or one-way analysis of variance with a Bonferroni correction. Data plotting was performed with GraphPad Prism, version 5 (GraphPad Software, Inc., La Jolla, CA, USA). P $<0.05$ was considered to indicate a statistically significant difference.

\section{Results}

miR-144 expression is downregulated in cervical cancer tissues. To assess the function of miR-144 in cervical cancer, the present study first examined its expression in the cervical tissues from patients with cervical cancer. The RT-qPCR results demonstrated that miR-144 expression was significantly reduced in the cervical cancer tissues compared with matched normal cervical tissues (Fig. $1 \mathrm{~A} ; \mathrm{P}<0.01$ ).

A previous miRNA microarray analysis reported that miR-144 expression was downregulated in patients with lymph node metastasis (21). Therefore, the present study also detected the expression levels of miR-144 in patients with lymph node metastasis. As presented in Fig. 1B, miR-144 expression in cervical cancer tissues of patients with lymph node metastasis was also lower than that in patients without lymph node metastasis $(\mathrm{P}<0.05)$, implying that decreased expression of miR-144 may have an important role in the progression of cervical cancer.

Association of miR-144 expression with clinicopathological features of cervical cancer patients. In order to determine the clinical significance of miR-144 in cervical cancer, the 35 patients were divided into two groups based on miR-144 expression levels (low vs. high) with the median expression level as a cut-off point. The association of miR-144 expression with the clinicopathological features is presented in Table I. The results indicated that downregulation of miR-144 was significantly associated with the FIGO stage $(\mathrm{P}=0.004)$ and lymph node metastasis $(\mathrm{P}=0.007)$, while no significant association with any other clinicopathological variables was observed.

Ectopic expression of miR-144 inhibits the proliferation of cervical cancer cells. To identify the role of miR-144 in cervical cancer cells, miR-144 mimics or NC was introduced into HeLa and C33A cells. The proliferation of miR-144-overexpressing cells was evaluated by an MTT assay. Transfection 

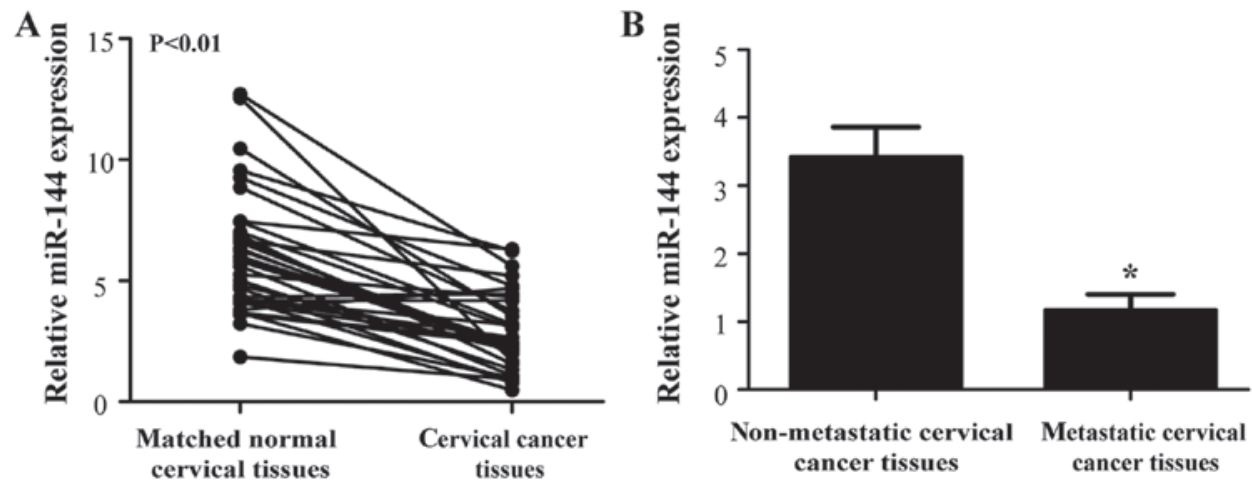

Figure 1. miR-144 expression is downregulated in cervical cancer tissues. (A) Expression of miR-144 was detected by reverse-transcription quantitative polymerase chain reaction analysis in cervical cancer tissues and matched normal tissues. (B) The levels of miR-144 in cancer tissues from patients with lymph node metastasis was lower compared with patients without lymph node metastasis. " $\mathrm{P}<0.05$ compared with non-metastatic cervical cancer tissues. miR, microRNA.

A

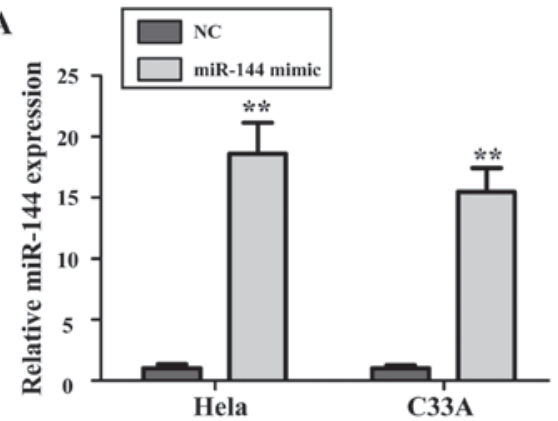

B

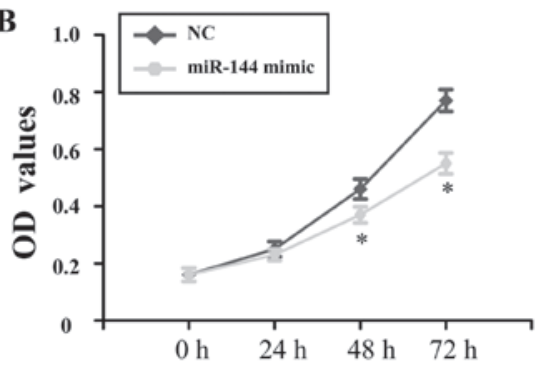

C

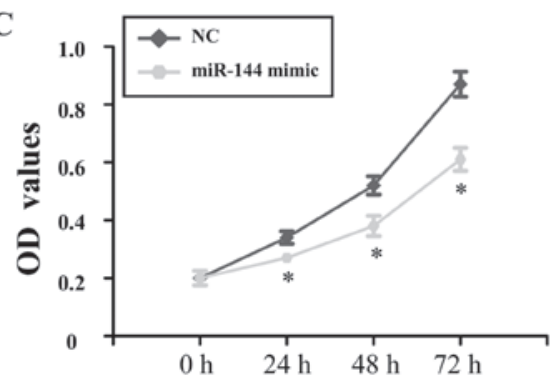

Figure 2. Ectopic expression of miR-144 inhibited the proliferation of cervical cancer cells. (A) Reverse-transcription quantitative polymerase chain reaction analysis of miR-144 levels in HeLa and C33A cells transfected with miR-144 mimics or NC. (B and C) Identification of cell growth by an MTT assay. (B) HeLa and (C) C33A cells. ${ }^{*} \mathrm{P}<0.05,{ }^{* *} \mathrm{P}<0.01$ and compared with NC. NC, negative control; OD, optical density; miR, microRNA.

with miR-144 mimics resulted in a significantly increased expression of miR-144 in HeLa and C33A ( $\mathrm{P}<0.01$; Fig. 2A). The results of the MTT assay demonstrated that at 48 and $72 \mathrm{~h}$ after transfection with miR-144 overexpression vector, the growth of HeLa and C33A cells was significantly inhibited compared with that in the NC group ( $\mathrm{P}<0.05$; Fig. $2 \mathrm{~B}$ and $\mathrm{C})$.
Overexpression of miR-144 decreases the migration and invasion abilities of cervical cancer cells. Next, the present study sought to explore the effect of miR-144 on the migration and invasion abilities of cervical cancer cells. As presented in Fig. 3A, the migration abilities of HeLa and C33A cells treated with miR-144 mimics were significantly decreased compared with those in $\mathrm{NC}$-transfected cells $(\mathrm{P}<0.05)$. As presented in Fig. 3B, the number of invasive cells in the miR-144 mimics-transfected groups was reduced compared with that in the NC-transfected groups (HeLa, $(75.4 \pm 17.6$ vs. $267.6 \pm 44.2$, $\mathrm{P}<0.01 ; \mathrm{C} 33 \mathrm{~A}, 86.9 \pm 21.3$ vs. $315.2 \pm 69.8, \mathrm{P}<0.01)$.

miR-144 targets the 3'-UTRs of VEGFA and VEGFC $m R N A$ to inhibit their expression. To further illustrate the molecular mechanisms underlying the tumor suppressor role of miR-144 in cervical cancer cells, a bioinformatics analysis was performed to identify targets of miR-144. A TargetScan (http://www.targetscan.org) search revealed the presence of a miR-144 binding site in the 3'-UTR of VEGFA mRNA as well as that of VEGFC mRNA (Fig. 4A). To verify this, miR-144 mimics or NC and the WT or MUT VEGFA or VEGFC luciferase reporter vectors were co-transfected into HeLa cells. The results indicated that the relative luciferase activity in miR-144 mimics-treated HeLa cells was significantly lower than that in NC-transfected cells ( $\mathrm{P}<0.05$; Fig. 4B), whereas this inhibitory effect was abrogated when the oligonucleotides in the binding sites of the 3'-UTRs were mutated. To further confirm that VEGFA and VEGFC are the targets of miR-144, RT-qPCR and western blot analyses were performed. The expression of VEGFA and VEGFC in HeLa cells was markedly reduced following miR-144 overexpression ( $\mathrm{P}<0.05$; Fig. $4 \mathrm{C}$ and $\mathrm{D})$. These results suggested that VEGFA and VEGFC are target genes of miR-144 in cervical cancer cells.

\section{Discussion}

Previous studies have indicated that $99.7 \%$ of cases with cervical cancer may be attributed to HPV infection (24). Emerging evidence has demonstrated that certain aberrantly expressed miRNAs are associated with tumorigenesis by upregulating the expression of oncogenes and serve as biomarkers for the diagnosis and prognosis of various human cancer types, 

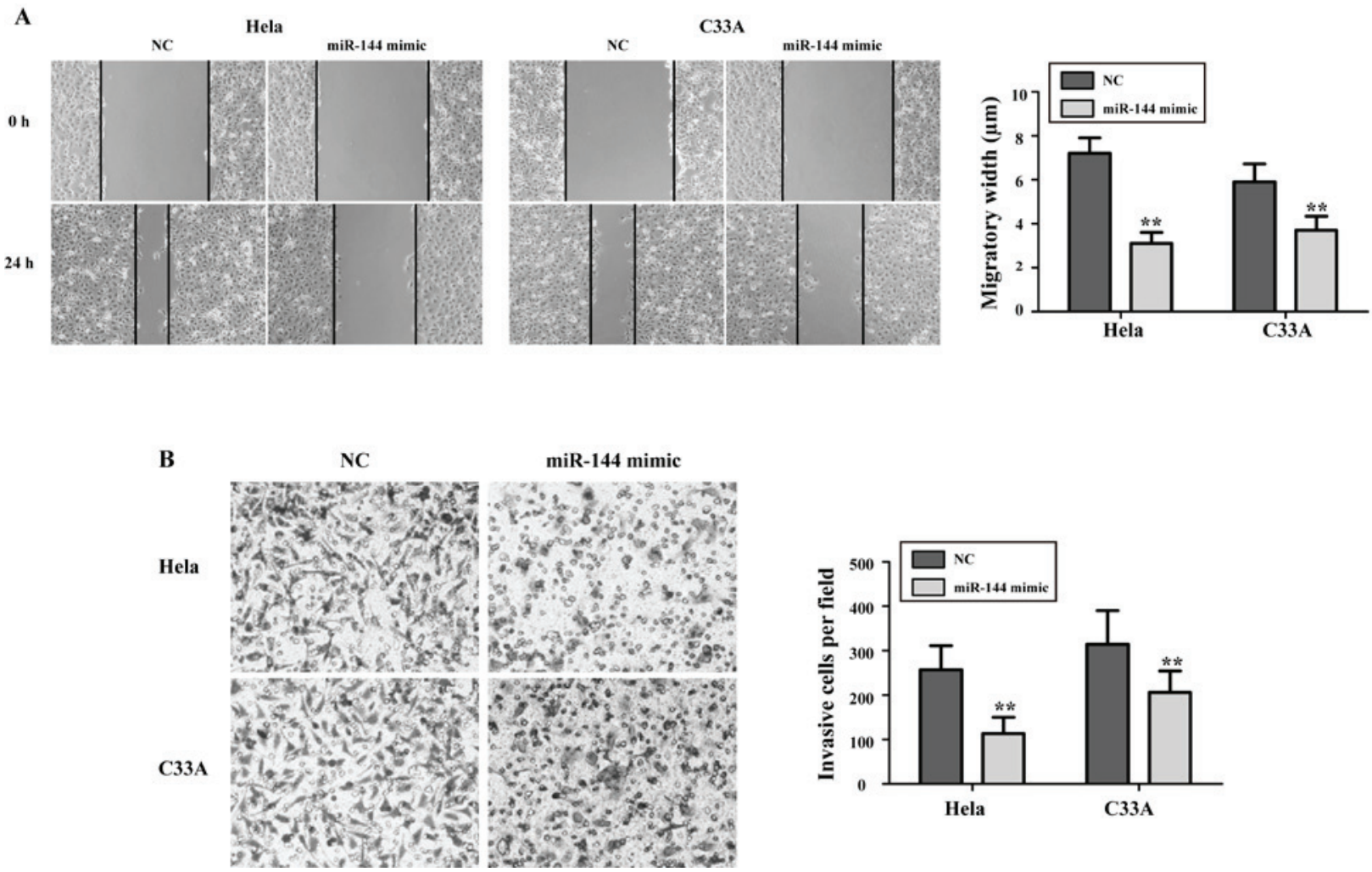

Figure 3. Overexpression of miR-144 decreases the migration and invasion abilities of cervical cancer cells. (A) Wound healing assay for assessment of cell migration. Representative photomicrographs of HeLa and C33A cells are displayed (magnification, x100). The black lines indicate the migration front. (B) Transwell assay for assessment of cell invasion. Representative photomicrographs of the lower side of the Transwell membranes are displayed (magnification, x200). Following transfection with miR-144 mimics, the number of invasive HeLa and C33A cells was significantly reduced compared with that in the NC group. ${ }^{* *} \mathrm{P}<0.01$ compared with NC. NC, negative control; miR, microRNA.
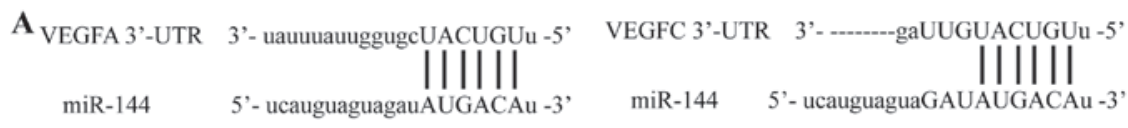

B
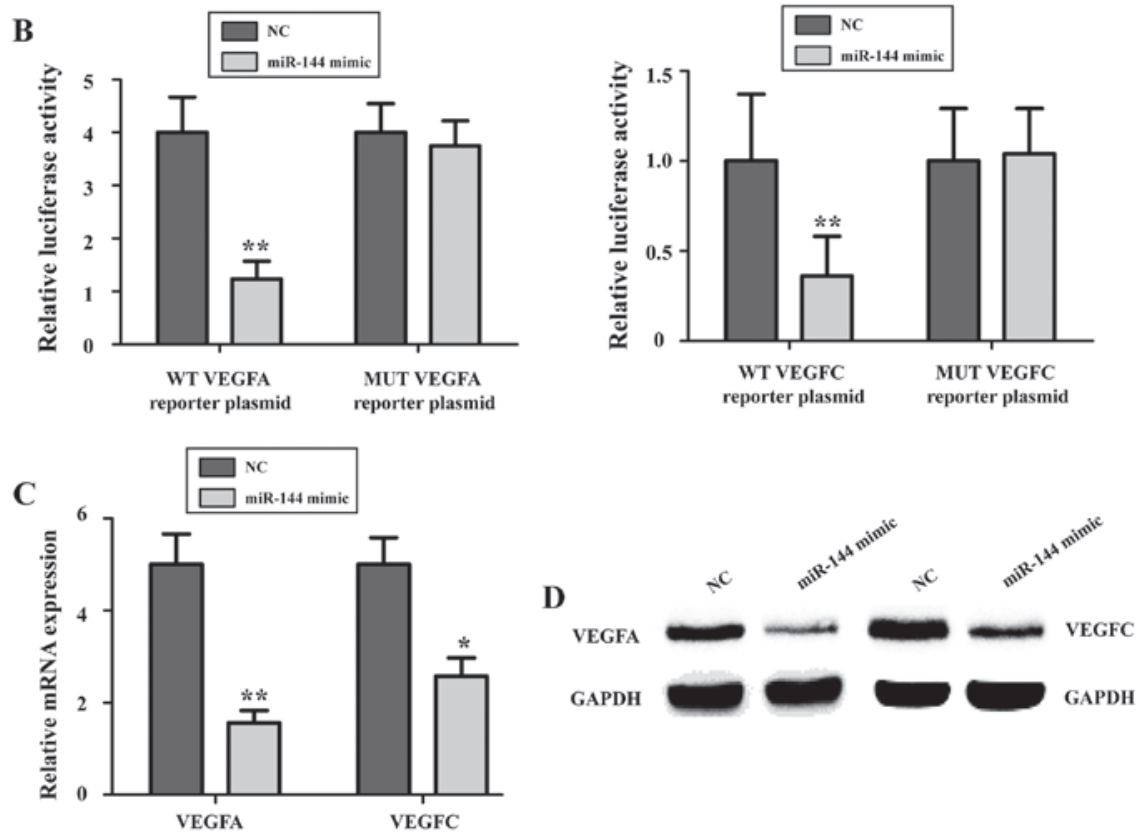

Figure 4. VEGFA and VEGFC are target genes of miR-144 in cervical cancer cells. (A) The binding sites of miR-144 on 3'-UTRs of VEGFA and VEGFC as predicted by a bioinformatics analysis. (B) The WT or MUT VEGFA or VEGFC reporter plasmids was co-transfected with miR-144 mimics or NC into HeLa cells. Luciferase activity was measured at $24 \mathrm{~h}$ after transfection. (C) Reverse-transcription quantitative polymerase chain reaction analysis and (D) western blot analysis of VEGFA and VEGFC expression in HeLa cells after transfection with miR-144 mimics or NC. "P<0.05 and " $\mathrm{*}<0.01$ compared with NC. VEGFA, vascular endothelial growth factor A; NC, negative control; miR, microRNA; WT, wild-type; MUT, mutated; UTR, untranslated region. 
including cervical cancer (25-27). Hence, identification of miRNAs and their target genes involved in tumorigenesis will provide key clues for the development of novel diagnostic tools and therapies for patients with cervical cancer.

miR-144 is located on chromosome 17q11.2 of the human genome, which has been reported to be downregulated in esophageal squamous cell cancer (28), laryngeal squamous cell carcinoma (29), lung cancer (30) and bladder cancer (31). The present study assessed the expression levels of miR-144 in cervical cancer tissues and their matched normal cervical tissues to determine the effects of miR-144 on the malignant progression of cervical cancer. The results indicated that miR-144 was markedly downregulated in cervical cancer tissues, which was consistent with those of previous studies. A miRNA microarray analysis by Ding et al (21) identified that the expression of miR-144 was significantly downregulated in cervical cancer patients with lymph node metastasis as compared with that in patients without. In line with this, the present study also indicated that the expression of miR-144 was lower in cervical cancer tissues from patients with lymph node metastasis compared with patients without lymph node metastasis. The downregulation of miR-144 was significantly associated with the FIGO stage and lymph node metastasis. These results strongly suggested that miR-144 is involved in the progression of cervical cancer.

Epigenetic changes represent an important mechanism for the silencing of tumor suppressor genes or activation of oncogenes. In cervical cancer, DNA methylation-mediated silencing of a rapidly growing number of coding and non-coding genes has been identified and their functional relevance has been demonstrated (32). Increased methylation has been demonstrated for a number of miRNAs, including miR-34b, miR-95, miR-124 and miR-375 (32). The present study found that miR-144 expression was also repressed, indicating that DNA methylation-mediated silencing of miR-144 may exist in cervical cancer.

miR-144 has been indicated to have a tumor suppressive role in the progression of cancer. For instance, Wang et al (33) identified that miR-144 suppressed osteosarcoma cell proliferation and metastasis by targeting the expression of Rho-associated protein kinases 1 and 2. Matsushita et al (34) suggested that tumor-suppressive miR-144 directly targeted the transcripts of CCNE1/2 as potential prognostic biomarkers in bladder cancer. Zhang et al (30) reported that miR-144-3p, a tumor suppressive miRNA, targeted ETS-1 in laryngeal squamous cell carcinoma. The present study expanded the current knowledge regarding the role of miR-144 in cervical cancer and suggested a tumor suppressive role via inhibition of cellular growth, migration and invasion.

Since miRNAs usually exert their biological effects via inhibiting the expression of target mRNAs, a bioinformatics analysis was performed to predict gene targets of miR-144. VEGFA and VEGFC were predicted as targets, which are two cancer-associated genes containing highly evolutionarily conserved miR-144 binding sites on the 3'-UTR of their mRNAs. A previous study reported that VEGF contributed to endothelial cell proliferation, division and migration (35). Knockdown of hypoxia-inducible factor- $1 \alpha$ was previously demonstrated to decrease the radiation resistance of cervical cancer by reducing the expression of VEGF (36). Anti-VEGF treatment augments the tumor radiation response under normoxic or hypoxic conditions, which is a novel therapeutic strategy in cervical cancer (37). VEGFA and VEGFC, two members of the VEGF family, were recently identified as the predominant tumor metastasis-driving factors in various human cancers types, including renal cell carcinoma (38), hepatocellular carcinoma (39) and cervical cancer (40-42). The present study identified VEGFA and VEGFC as targets of miR-144 via directly binding to the 3'-UTRs of VEGFA and VEGFC mRNA in a dual luciferase reporter assay. Furthermore, RT-qPCR and western blot analyses confirmed that ectopic expression of miR-144 significantly downregulated the expression of VEGFA and VEGFC. These findings suggested that downregulation of miR-144 may contribute to aberrant growth and metastasis of cancer cells through loss of inhibition of VEGFA and VEGFC expression.

In conclusion, the present study demonstrated that miR-144 exerts a suppressive effect on the growth, migration and invasion of cervical cancer cells by directly targeting VEGFA and VEGFC, indicating that miR-144 may be a promising therapeutic target for cervical cancer treatment.

\section{Acknowledgements}

The present study was supported by the Leader Training Plan of the Health Department, Shanghai Pudong New District (grant no. PWRd2013-09), the Key Specialty Construction Project of Pudong Health and Family Planning Commission of Shanghai (grant no. PWZz2013-14) and the Science and Technology Development Project of Pudong New District (grant no. PW2013A-6).

\section{References}

1. Siegel R, Ma J, Zou Z and Jemal A: Cancer statistics, 2014. CA Cancer J Clin 64: 9-29, 2014.

2. Jemal A, Bray F, Center MM, Ferlay J, Ward E and Forman D: Global cancer statistics. CA Cancer J Clin 61: 69-90, 2011.

3. Hildesheim A and Wang SS: Host and viral genetics and risk of cervical cancer: A review. Virus Res 89: 229-240, 2002.

4. Castellsagué X: Natural history and epidemiology of HPV infection and cervical cancer. Gynecol Oncol 110 (3 Suppl 2): S4-S7, 2008.

5. Du CX and Wang Y: Expression of P-Akt, NFkappaB and their correlation with human papillomavirus infection in cervical carcinoma. Eur J Gynaecol Oncol 33: 274-277, 2012.

6. Bartel DP: MicroRNAs: Target recognition and regulatory functions. Cell 136: 215-233, 2009.

7. Moreno-Moya JM, Vilella F and Simón C: MicroRNA: Key gene expression regulators. Fertil Steril 101: 1516-1523, 2014.

8. Ventura A and Jacks T: MicroRNAs and cancer: Short RNAs go a long way. Cell 136: 586-591, 2009.

9. Bartels CL and Tsongalis GJ: MicroRNAs: Novel biomarkers for human cancer. Clin Chem 55: 623-631, 2009.

10. Wang X, Tang S, Le SY, Lu R, Rader JS, Meyers C and Zheng ZM: Aberrant expression of oncogenic and tumor-suppressive microRNAs in cervical cancer is required for cancer cell growth. PLoS One 3: e2557, 2008.

11. Ambros V: The functions of animal microRNAs. Nature 431: 350-355, 2004.

12. Wang X and Xia Y: microRNA-328 inhibits cervical cancer cell proliferation and tumorigenesis by targeting TCF7L2. Biochem Biophys Res Commun 475: 169-175, 2016.

13. Ye H, Yu X, Xia J, Tang X, Tang L and Chen F: MiR-486-3p targeting ECM1 represses cell proliferation and metastasis in cervical cancer. Biomed Pharmacother 80: 109-114, 2016.

14. Zhou JY, Zheng SR, Liu J, Shi R, Yu HL and Wei M: MiR-519d facilitates the progression and metastasis of cervical cancer through direct targeting Smad7. Cancer Cell Int 16: 21, 2016. 
15. Cheng YX, Zhang QF, Hong L, Pan F, Huang JL, Li BS and Hu M: MicroRNA-200b suppresses cell invasion and metastasis by inhibiting the epithelial-mesenchymal transition in cervical carcinoma. Mol Med Rep 13: 3155-3160, 2016.

16. Dore LC, Amigo JD, Dos Santos CO, Zhang Z, Gai X, Tobias JW Yu D, Klein AM, Dorman C, Wu W, et al: A GATA-1-regulated microRNA locus essential for erythropoiesis. Proc Natl Acad Sci USA 105: 3333-3338, 2008.

17. Lawrie $\mathrm{CH}$ : microRNA expression in erythropoiesis and erythroid disorders. Br J Haematol 150: 144-151, 2010.

18. Cao T, Li H, Hu Y, Ma D and Cai X: miR-144 suppresses the proliferation and metastasis of hepatocellular carcinoma by targeting E2F3. Tumour Biol 35: 10759-10764, 2014.

19. Cai SD, Chen JS, Xi ZW, Zhang LJ, Niu ML and Gao ZY: MicroRNA144 inhibits migration and proliferation in rectal cancer by downregulating ROCK-1. Mol Med Rep 12: 7396-7402, 2015.

20. Wang H, Wang A, Hu Z, Xu X, Liu Z and Wang Z: A Critical role of miR-144 in diffuse large B-cell lymphoma proliferation and invasion. Cancer Immunol Res 4: 337-344, 2016.

21. Ding H, Wu YL, Wang YX and Zhu FF: Characterization of the microRNA expression profile of cervical squamous cell carcinoma metastases. Asian Pac J Cancer Prev 15: 1675-1679, 2014.

22. Du L, Lei Y, Li D, Qiu X and Liang B: Value of magnetic resonance imaging in preoperative staging of endometrial carcinoma according to International Federation of Gynecology and Obstetrics (2009) staging criteria. Nan Fang Yi Ke Da Xue Xue Bao 32: 1048-1051, 2012 (In Chinese).

23. Schmittgen TD and Livak KJ: Analyzing real-time PCR data by the comparative C(T) method. Nat Protoc 3: 1101-1108, 2008.

24. Wang LQ, Zhang Y, Yan H, Liu KJ and Zhang S: MicroRNA-373 functions as an oncogene and targets YOD1 gene in cervical cancer. Biochem Biophys Res Commun 459: 515-520, 2015.

25. Schickel R, Boyerinas B, Park SM and Peter ME: MicroRNAs: Key players in the immune system, differentiation, tumorigenesis and cell death. Oncogene 27: 5959-5974, 2008.

26. Chen B, Hou Z, Li C and Tong Y: MiRNA-494 inhibits metastasis of cervical cancer through Pttg1. Tumour Biol 36: 7143-7149, 2015.

27. Wang N, Wei H, Yin D, Lu Y, Zhang Y, Zhang Q, Ma X and Zhang S: MicroRNA-195 inhibits proliferation of cervical cancer cells by targeting cyclin D1a. Tumour Biol 37: 4711-4720, 2016.

28. Shao Y, Li P, Zhu ST, Yue JP, Ji XJ, Ma D, Wang L, Wang YJ, Zong Y, Wu YD and Zhang ST: MiR-26a and miR-144 inhibit proliferation and metastasis of esophageal squamous cell cancer by inhibiting cyclooxygenase-2. Oncotarget 7: 15173-15186, 2016.

29. Wu X, Cui CL, Chen WL, Fu ZY, Cui XY and Gong X: miR-144 suppresses the growth and metastasis of laryngeal squamous cell carcinoma by targeting IRS1. Am J Transl Res 8: 1-11, 2016.
30. Zhang SY, Lu ZM, Lin YF, Chen LS, Luo XN, Song XH, Chen SH and Wu YL: miR-144-3p, a tumor suppressive microRNA targeting ETS-1 in laryngeal squamous cell carcinoma. Oncotarget 7: 11637-11650, 2016.

31. Guo Y, Ying L, Tian Y, Yang P, Zhu Y, Wang Z, Qiu F and Lin J: miR-144 downregulation increases bladder cancer cell proliferation by targeting EZH 2 and regulating Wnt signaling. FEBS J 280: 4531-4538, 2013.

32. Wilting SM, Miok V, Jaspers A, Boon D, Sørgård H, Lando M, Snoek BC, van Wieringen WN, Meijer CJ, Lyng H, et al: Aberrant methylation-mediated silencing of microRNAs contributes to HPV-induced anchorage independence. Oncotarget 7: 43805-43819, 2016.

33. Wang W, Zhou X and Wei M: MicroRNA-144 suppresses osteosarcoma growth and metastasis by targeting ROCK1 and ROCK2. Oncotarget 6: 10297-10308, 2015.

34. Matsushita R, Seki N, Chiyomaru T, Inoguchi S, Ishihara T, Goto Y, Nishikawa R, Mataki H, Tatarano S, Itesako T, et al: Tumour-suppressive microRNA-144-5p directly targets CCNE1/2 as potential prognostic markers in bladder cancer. $\mathrm{Br} \mathrm{J}$ Cancer 113: 282-289, 2015.

35. Goel HL and Mercurio AM: VEGF targets the tumour cell. Nat Rev Cancer 13: 871-882, 2013.

36. Fu Z, Chen D, Cheng H and Wang F: Hypoxia-inducible factor-1 $\alpha$ protects cervical carcinoma cells from apoptosis induced by radiation via modulation of vascular endothelial growth factor and p53 under hypoxia. Med Sci Monit 21: 318-325, 2015.

37. Tsuda N, Watari H and Ushijima K: Chemotherapy and molecular targeting therapy for recurrent cervical cancer. Chin J Cancer Res 28: 241-253, 2016.

38. Cai Y, Li H and Zhang Y: Downregulation of microRNA-206 suppresses clear cell renal carcinoma proliferation and invasion by targeting vascular endothelial growth factor A. Oncol Rep 35: 1778-1786, 2016

39. Liu Z, Wang J, Mao Y, Zou B and Fan X: MicroRNA-101 suppresses migration and invasion via targeting vascular endothelial growth factor-C in hepatocellular carcinoma cells. Oncol Lett 11: 433-438, 2016.

40. Chen L, Wu YY, Liu P, Wang J, Wang G, Qin J, Zhou J and Zhu J: Down-regulation of HPV18 E6, E7, or VEGF expression attenuates malignant biological behavior of human cervical cancer cells. Med Oncol 28 (Suppl 1): S528-S539, 2011.

41. Liu J, Cheng Y, He M and Yao S: Vascular endothelial growth factor $C$ enhances cervical cancer cell invasiveness via upregulation of galectin-3 protein. Gynecol Endocrinol 30: 461-465, 2014.

42. Chen H, Suo K, Cheng Y, Zheng B and Xu L: Vascular endothelial growth factor $\mathrm{C}$ enhances cervical cancer migration and invasion via activation of focal adhesion kinase. Gynecol Endocrinol 29: 20-24, 2013. 